\title{
Radar-based Mapping of the Environment: Occupancy Grid-Map vs SAR
}

\author{
Timo Grebner, Pirmin Schoeder, Vinzenz Janoudi, and Christian Waldschmidt
} resale or redistribution to servers or lists, or reuse of any copyrighted component of this work in other works. 


\title{
Radar-based Mapping of the Environment: Occupancy Grid-Map vs SAR
}

\author{
Timo Grebner, Graduate Student Member, IEEE, Pirmin Schoeder, Graduate Student Member, IEEE, \\ Vinzenz Janoudi, and Christian Waldschmidt, Senior Member, IEEE
}

\begin{abstract}
For autonomous driving vehicles highly accurate representations of the environment are essential for both trajectory planning and self-localization. Different possibilities allow to generate detailed maps of the environment based on chirpsequence radar sensors for advanced driver assistance systems (ADAS). For the first time, this article shows a qualitative comparison between synthetic aperture radar (SAR) and occupancy grid map (OGM) based environment representation using identical measurement data. The differences of existing signal processing chains as well as a visual measurement-based comparison of the resulting environmental maps is presented.
\end{abstract}

Index Terms-grid maps (GM), synthetic aperture radar (SAR), networks, ego-motion, radar systems, frequency modulated continuous wave (FMCW), mapping.

\section{INTRODUCTION}

$\mathbf{T}$ $\mathrm{HE}$ increasing progress in the field of autonomous driving leads to ever higher demands on the sensor systems. With the increasing complexity of autonomous driving applications, more accurate and detailed images of the environment are required. Radar sensors offer lower costs and higher robustness in comparison to optical based sensor systems [1]. The advantages of radar sensors stimulate the ever increasing use of radar sensors in the automotive sector [2]. In order to generate high-resolution environmental maps, new high-performance sensor architectures can be created or more information can be extracted from existing radar systems through effective signal processing [3]. The best known approach for mapping the environment with help of radar data are grid maps (GM), which can be classified into target list-based occupancy grid maps (OGM) and raw data-based synthetic aperture radar (SAR) maps. Different algorithms in order to generate radarbased grid maps have been presented in [4], [5], [6].

This paper provides for the first time a qualitative comparison between radar-based OGM and SAR maps for creating GMs based on identical raw data using existing mapping algorithms. Especially the scenarios in which either OGMor SAR-based mapping is advantageous, are highlighted. In chapter II, the basic known structures for generating GMs and for creating SAR images are presented. The measurement setup and the measurement-based comparison are described in chapters III and IV. Chapter V summarises the work.

The authors are with the Institute of Microwave Engineering, Ulm University, 89081 Ulm, Germany (e-mail: timo.grebner@uni-ulm.de).

Manuscript received April 19, 2021; revised August 16, 2021.

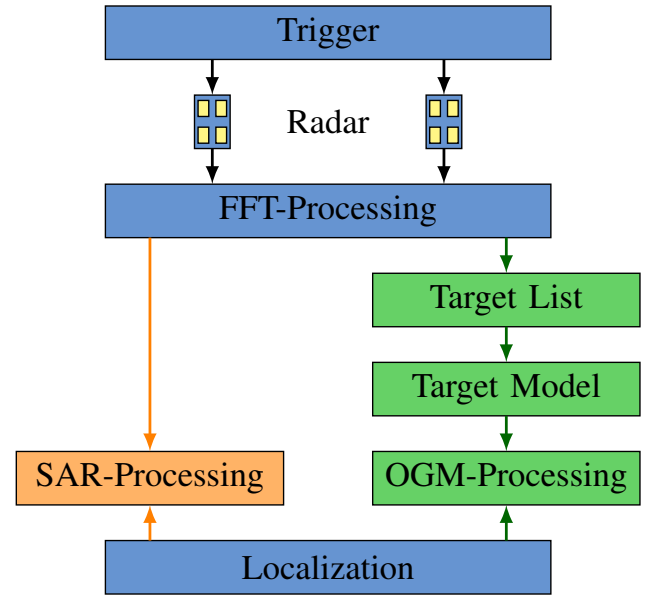

Fig. 1. Overview of the processing steps for the SAR- and OGM-based imaging based on the measurement data of $n=2$ radar sensors.

\section{PROCESSING CHAIN}

An overview of the entire signal processing is shown in Fig. 1. The raw data of $n$ externally triggered radar sensors is stored, processed independently, and transformed into the frequency domain using a Fast Fourier Transform (FFT). After the transformation into the frequency domain, a distinction between SAR processing and the generation of OGMs is made. Both OGMs and SAR images are generally cell-based representations of the environment. For ADAS using GMs to represent the environment, the distinction between occupied cells and free cells is of great relevance. OGMs and SAR images differ mainly in the resolution and the information content of each cell, but also in the signal processing of these maps. The quality of the resulting maps depends significantly on the localization and orientation accuracy of the vehicle, which is determined with high precision for the following measurement with help of a tachymeter [7].

\section{A. Occupancy Grid Map}

OGMs are generally target list-based mapping algorithms [8]. A distinction is usually made between amplitude and occupancy grid maps, whereby the probabilistic OGM has clear robustness advantages [5]. For the generation of OGMs, the target lists are generated based on the range-Doppler information, calculated by applying a 2D-FFT on the ADC samples [9]. The relevant target information is extracted by 


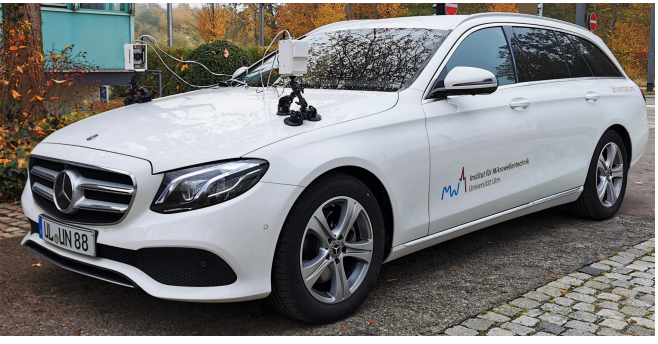

Fig. 2. Experimental system setup with two side-look radar sensors on a car.

using a constant false alarm rate (CFAR), subsequent peak detection, and angle of arrival (AoA) estimation. Based on these target lists, a target model as well as a sensor model is generated for each frame and each sensor [5], which can ultimately be used to generate an OGM and to update it frame by frame with [10]

$$
\begin{aligned}
& \frac{p\left(m_{i} \mid z_{1: \mathrm{t}}, x_{1: \mathrm{t}}\right)}{p\left(-m_{i} \mid z_{1: \mathrm{t}}, x_{1: \mathrm{t}}\right)} \\
& =\frac{p\left(m_{i} \mid z_{\mathrm{t}}, x_{\mathrm{t}}\right)}{\underbrace{1-p\left(m_{i} \mid z_{\mathrm{t}}, x_{\mathrm{t}}\right)}_{\text {current measurement }}} \underbrace{\frac{p\left(m_{i} \mid z_{1: \mathrm{t}-1}, x_{1: \mathrm{t}-1}\right)}{1-p\left(m_{i} \mid z_{1: \mathrm{t}-1}, x_{1: \mathrm{t}-1}\right)}}_{\text {recursive term }} \underbrace{\frac{1-p\left(m_{i}\right)}{p\left(m_{i}\right)}}_{\text {a priori information }} .
\end{aligned}
$$

Whereby $p\left(m_{i} \mid z_{1: \mathrm{t}}, x_{1: \mathrm{t}}\right)$ describes the occupancy probability of the $i$-th cell at time $t, x$ the pose of the radar sensor and $z$ the measurement data. This allows a map update exclusively based on the current measurement including a priori information and the currently existing map.

\section{B. SAR Image}

In contrast to OGMs, the time-domain samples are used after applying a 1D-FFT directly for the generation of SAR maps, for which reason no target lists and no probabilistic modelling is necessary. For comparison purpose, the backprojection algorithm (BP) is used, which calculates the power of the cell $m_{i}$ depending on the given sensor position $x$ and sensor measurement $z$. The associated complex values of the range measurements $A_{r, k}$ are coherently projected to each grid cell resulting in complex amplitudes $G$ [11], [12], [13]

$$
\begin{aligned}
& G\left(m_{i} \mid z_{1: \mathrm{t}}, x_{1: \mathrm{t}}\right)=G\left(m_{i} \mid z_{1: \mathrm{t}-1}, x_{1: \mathrm{t}-1}\right) \\
& +\sum_{k=1}^{K} A_{r, k}\left(m_{i} \mid z_{\mathrm{t}_{\mathrm{k}}}\right) \cdot \exp \left(-\mathrm{j} 2 \pi \frac{f_{\text {start }}}{c_{0}} \cdot 2 r\left(m_{i} \mid x_{\mathrm{t}_{\mathrm{k}}}\right)\right) .
\end{aligned}
$$

To ensure a proper comparison, frame-based radar sensors are used for the evaluation, whereby $t$ is discretised to the frame rate, $t_{k}$ represents the time stamps of the individual $K$ ramps, and $r$ is the distance. In contrast to OGM, SAR does not accumulate probabilities $p$ in each cell but complex amplitudes $G$ representing the reflected power.

\section{Measurement Setup}

The measurement setup used for evaluating the presented methods is shown in Fig. 2 and consists of two incoherently
TABLE I

USED RADAR PARAMETERS FOR GRID-MAP AND SAR-PROCESSING.

\begin{tabular}{l|c|c} 
Parameter & \multicolumn{2}{|c}{ Value } \\
\hline Start frequency $f_{\text {start }}$ & $S_{1}$ & \multicolumn{2}{c}{$S_{2}$} \\
Bandwidth $B$ & $77.6 \mathrm{GHz}$ & $77.63 \mathrm{GHz}$ \\
\cline { 2 - 2 } Ramp repetition time $T_{\mathrm{r}}$ & \multicolumn{2}{|c}{$3.07 \mathrm{GHz}$} \\
Sampling frequency $f_{\mathrm{s}}(\mathrm{IQ})$ & \multicolumn{2}{|c}{$67 \mathrm{\mu s}$} \\
Number of ramps & \multicolumn{2}{|c}{384} \\
\hline Number of transmitters & 1 \\
Number of receivers & 4 \\
\hline Measurement rate & \multicolumn{2}{|c}{$37 \mathrm{~Hz}$}
\end{tabular}

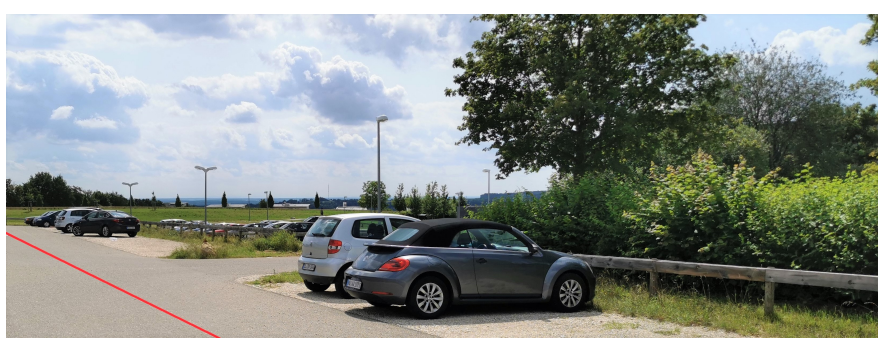

Fig. 3. Photograph of the measurement scene with several parked cars, hedges and trees, fences, roads, and gravel car parks. Approximated trajectory in red.

operated distributed chirp sequence radar sensors. A trigger is used to synchronise the radar sensors in the network to avoid interference. A small shift in the carrier frequency of the radar sensors prevents interference between them. The sensors are arranged in such a way that the lateral areas, relevant for SAR, are illuminated, and a large field of view (FoV) is covered. The radar parameters are listed in Table I.

The measurement was performed along a rectilinear trajectory $((0,0) \rightarrow(128,0)) \mathrm{m}$ with a speed up to $15 \mathrm{~m} \mathrm{~s}^{-1}$ on a parking lot, where numerous parked cars as well as other obstacles such as fences, lanterns, and trees exist as shown in Fig. 3.

\section{Measurement Results}

For reasons of comparability, the same measurement data were used to calculate OGM- and SAR-based maps with a pixel size of $1 \mathrm{~cm}$. The result after SAR processing is shown in Fig. 4a, the result of OGM in Fig. 4b. Significant differences in detectability, resolution and identifiability can be observed, as explained in the following section.

\section{A. Detectability}

Targets with a high RCS, such as street lamps or cars, as well as low radar cross section (RCS) targets with large surface area, such as hedges and trees, can be clearly detected in both representations and distinguished from the free space. This is in contrast to low RCS targets with small surface areas such as the fence, which is only visible in the SAR-based image.

In the SAR image, the Fence 1 and Fence 2 can be recognised as a continuous obstacle, whereas in the OGM only the posts of the fence are visible. These wrongly detected free spaces of about $2 \mathrm{~m}$ can be problematic and lead to unsafe trajectory planning. Here, the SAR processing of the raw data clearly provides robustness advantages. 

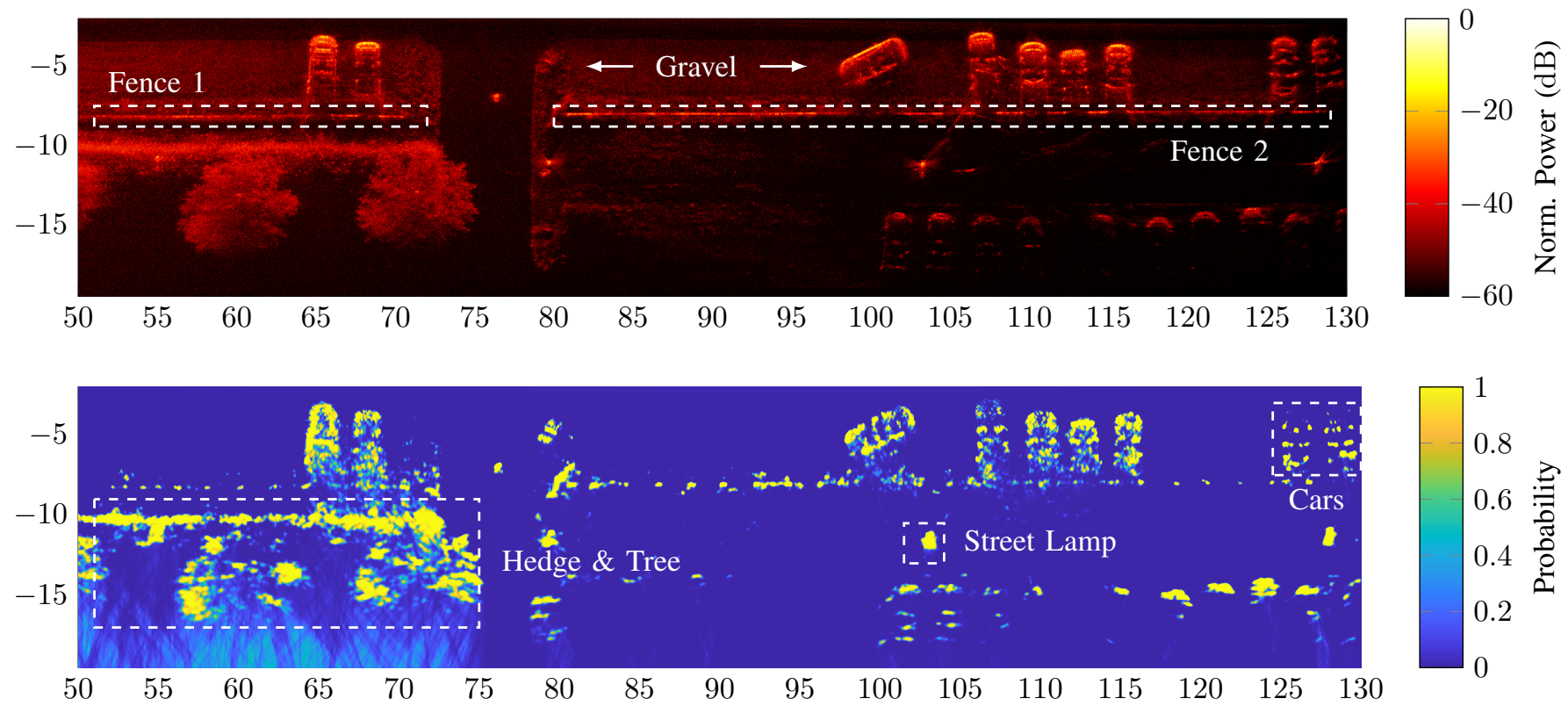

Fig. 4. Comparison of SAR-based (top) and OGM-based (bottom) mapping methods of a car park with vehicles, street lights, hedges and trees, and fences. $x$ and $y$ axis in $\mathrm{m}$.

\section{B. Resolution and Accuracy}

The contour of all targets in the SAR image is clearly more detailed and focussed. The individual branches of the trees as well as the vehicle contours can be mapped more precisely, which is especially valid for targets with many scattering centres. Therefore, even individual branches of the trees can thus be captured in detail, also inside the tree crown.

In addition, the resolution strongly depends on the synthetic aperture size, which can be observed from the marked cars. At the end of the trajectory at $(128,0) \mathrm{m}$ and thus the reduced synthetic aperture, the cars marked in the OGM are mapped worse and can no longer be identified or clustered as such, while for the SAR image, a short aperture is sufficient to map them in detail.

\section{Additional Information}

Due to different processing steps, the SAR image is powerdependent, which results in objects at a greater distance and lower radar cross section (RCS) being represented more faintly. As soon as it is a matter of identifying surrounding areas, such as asphalt or gravel, this is only possible to a limited extent with an OGM due to the probabilistic state description. While the OGM represents all grounds, such as asphalt or gravel, as "Free-Space", the SAR image can clearly resolves between the road and the gravel path on which the vehicles are parked.

Likewise, the resolution of the OGM is not sufficiently high to depict objects in detail, which can be seen in Fig. 5 (top and bottom). While only point clouds are recognizable in the OGM, the contour of the car can be identified visually in the SAR image. Furthermore, Fig. 5a shows that only one car is parked with the cowl facing forwards, as both the side mirrors and the rear-view mirror can be seen.
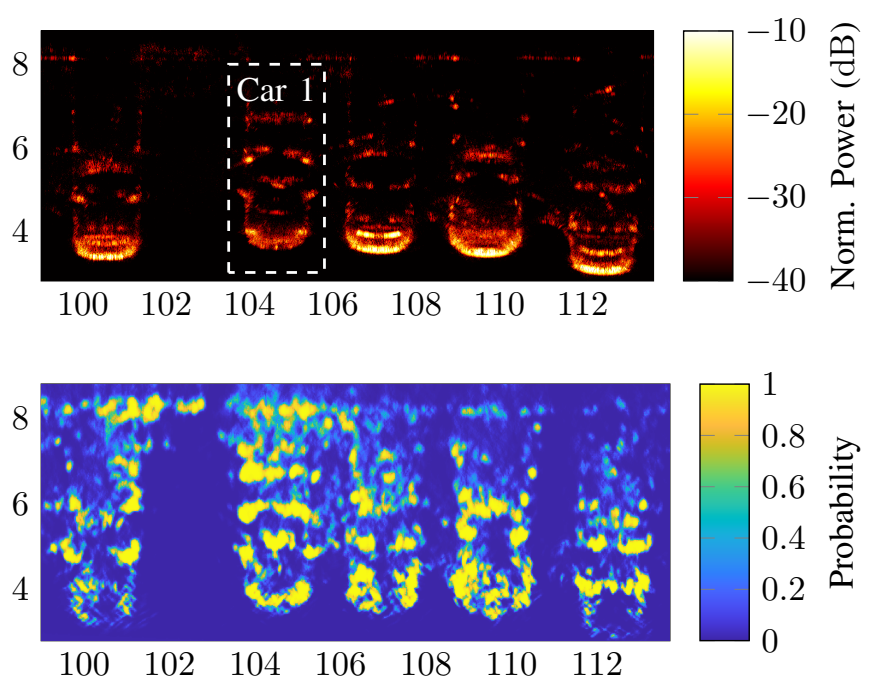

Fig. 5. Detailed comparison of parked cars for SAR-processed image (top) and OGM-based representation (bottom). $x$ and $y$ axis in $\mathrm{m}$.

\section{CONClusion}

For the first time, a qualitative comparison between SARand OGM-based mapping results could be made based on identical measurement data. The advantages of OGM-based maps are mainly the robustness with respect to false detections and measurement inaccuracies. Moreover, the occupancy status of each cell is directly determinable from the cell amplitude. SAR mapping is much more computationally expensive due to the ramp-by-ramp update step. However, SAR provides much more accurate images of the environment, which ultimately enables the detection of even small details as well as street conditions. Moreover, due to raw data processing, SAR images are independent of pre-segmented target lists. 


\section{REFERENCES}

[1] F. Roos, J. Bechter, C. Knill, B. Schweizer, and C. Waldschmidt, "Radar sensors for autonomous driving: Modulation schemes and interference mitigation," IEEE Microw. Mag., vol. 20, pp. 58-72, 2019.

[2] C. Waldschmidt, J. Hasch, and W. Menzel, "Automotive radar - from first efforts to future systems," IEEE J. Microw., vol. 1, pp. 135-148, 2021.

[3] M. Gottinger, M. Hoffmann, M. Christmann, M. Schütz, F. Kirsch, P. Gulden, and M. Vossiek, "Coherent automotive radar networks: The next generation of radar-based imaging and mapping," IEEE J. Microw., vol. 1, no. 1, pp. 149-163, 2021.

[4] K. Werber, M. Rapp, J. Klappstein, M. Hahn, J. Dickmann, K. Dietmayer, and C. Waldschmidt, "Automotive radar gridmap representations," in IEEE MTT-S Int. Conf. Microw. Intell. Mobil. (ICMIM), 2015, pp. 1-4.

[5] P. Hügler, T. Grebner, C. Knill, and C. Waldschmidt, "Uav-borne 2-d and 3-d radar-based grid mapping," IEEE Geosci. Remote Sens. Lett., pp. $1-5,2020$.

[6] M. Steiner, T. Grebner, and C. Waldschmidt, "Millimeter-wave sarimaging with radar networks based on radar self-localization," IEEE Trans. Microw. Theory Techn., vol. 68, no. 11, pp. 4652-4661, 2020.

[7] Trimble Universale Totalstation, 2018.

[8] S. Thrun, "Learning metric-topological maps for indoor mobile robot navigation," Artif. Intell., vol. 99, no. 1, pp. 21-71, 1998.

[9] V. Winkler, "Range Doppler detection for automotive FMCW radars," in Eur. Microw. Conf., Oct. 2007, pp. 1445-1448.

[10] S. Thrun, "Learning occupancy grids with forward models," in IEEE Int. Conf. Intell. Robots Syst., vol. 3, 2001, pp. 1676-1681 vol.3.

[11] J. C. Curlander and R. N. Mcdonough, "Synthetic aperture radar: Systems and signal processing," 1991.

[12] O. Frey, C. Magnard, M. Ruegg, and E. Meier, "Focusing of airborne synthetic aperture radar data from highly nonlinear flight tracks," IEEE Trans. Geosci. Remote Sens., vol. 47, no. 6, pp. 1844-1858, 2009.

[13] L. Ulander, H. Hellsten, and G. Stenstrom, "Synthetic-aperture radar processing using fast factorized back-projection," IEEE Trans. Aerosp. Electron. Syst., vol. 39, no. 3, pp. 760-776, 2003. 